\title{
Uso de la correlación y la regresión lineal para ampliar registros de volúmenes escurridos anuales
}

\author{
D.F. Campos-Aranda \\ Facultad de Ingeniería, UASLP \\ Email:dcampos@deimos.tc.uaslp.mx
}

(recibido: febrero de 1998; aceptado: septiembre de 1998)

Resumen

\begin{abstract}
Inicialmente se destaca la importancia de las estimaciones de la media y la desviación estándar como parámetros estadísticos básicos, los cuales caracterizan la magnitud y la variabilidad de los volúmenes escurridos anuales en los estudios hidrológicos de planeación del aprovechamiento de los recursos hidráulicos de una región. Enseguida, se describen con detalle las ecuaciones que permiten evaluar cuantitativamente si es conveniente o no, desde un punto de vista estadístico, ampliar el registro corto de escurrimientos, con base en datos comunes, adicionales y disponibles; esto en una o dos estaciones hidrométricas cercanas, con las cuales, el registro reducido guarda cierta correlación (dependencia o asociación). Lo anterior, significa evaluar si con base en el registro ampliado, las estimaciones de la media y la variancia mejoran estadísticamente. Posteriormente, se realizan dos aplicaciones numéricas a casos reales; una, para el modelo bidimensional que utiliza una estación hidrométrica cercana para ampliar el registro corto; y la otra, para el modelo tridimensional, que emplea dos estaciones de aforo auxiliares. Por último, se citan tres observaciones generales relativas al trabajo.
\end{abstract}

\begin{abstract}
Firstly, the estimates of standard deviation and arithmetic mean as basic statistical parameters are emphasised, which point out the variability and magnitude of annual streamflow records in the hydrological studies of planning water-resource developments inside a region. Then the equations for quantitative evaluations of statistical convenience of extending a short streamflow record are described in detail. The previous makes use of additional and common data in one or two closer hydrometric stations, with these the short observed record has a certain correlation (dependence or association). Later two numerical applications to real problems are given, the first one for the two dimensional model, which use a one close hydrometric station in order to extend the short record, and the second one application for the three dimensional model which makes use of two auxiliary hydrometric stations. Lastly, three general observations about the paper are cited.
\end{abstract}

\section{Introducción}

Los parámetros estadísticos básicos, la media $(X m)$, la desviación estándar $(S)$ y el coeficiente de variación $(C v=$ $S / \mathrm{Xm})$, permiten especificar las características del escurrimiento anual; el primero, en relación con su magnitud promedio y los segundos, respecto a sus características de dispersión o variabilidad. Por ello, tales parámetros son de uso común en la planeación del aprovechamiento de los recursos hidráulicos.
Estos parámetros son usualmente estimados con base en los registros disponibles de volúmenes escurridos anuales, de manera que si el registro es corto, los parámetros evaluados pueden tener poca garantía o cercanía a los valores poblacionales. Por lo anterior, y bajo ciertas circunstancias, la correlación con datos de otros sitios o estaciones hidrométricas cercanas puede ser empleada para mejorar las estimaciones de los parámetros en sitios o localidades con registros reducidos. El uso de la regresión lineal es la técnica básica para ampliar un registro corto con base en 
los datos de otro cercano con el cual se tiene cierta dependencia o asociación (correlación).

Por otra parte, cuando se desea asignar probabilidades a las estimaciones del potencial hidráulico o hidrológico de un río, es común emplear distribuciones de probabilidad del escurrimiento anual, las cuales pueden ser las de los modelos Normal, Log-normal o de Weibull, cuyas características cuantitativas son definidas a través de los parámetros estadísticos citados.

Por lo tanto, resulta conveniente investigar si la ampliación de un registro corto con base en la información común y adicional disponible en una o dos estaciones hidrométricas cercanas es conveniente desde un punto de vista estadístico, esto es, si mejora la estimación de los parámetros estadísticos, base de futuras evaluaciones asociadas con el potencial hidrológico de la corriente y con el desarrollo futuro de los recursos hidráulicos de una zona o región.

\section{Repaso de conceptos estadísticos básicos}

\section{Media y variancia}

La media de una muestra de $N$ términos, cada uno de los cuales tiene un valor numérico $\mathrm{X}_{i}$, está dada por

$$
\begin{gathered}
\text { Media }=\mathrm{Xm}=(1 / \mathrm{N}) \\
\left(\mathrm{X}_{1}+\mathrm{X}_{2}+\cdots \cdots \cdots+\mathrm{X}_{\mathrm{N}}\right)=(1 / \mathrm{N}) \Sigma \mathrm{X}_{\mathrm{i}}
\end{gathered}
$$

donde, la sumatoria abarca de 1 a $N$. La media aritmética es una cuantificación del promedio o tendencia central de los datos. La media de la población es comúnmente desig. nada por $\mu$.

En cambio, la variancia de una muestra es una medida del grado de dispersión de los términos alrededor de su valor medio y por ello se define como:

$$
\text { Variancia }=\operatorname{Var}(\mathrm{X})=(1 / \mathrm{N}) \Sigma\left(\mathrm{X}_{\mathrm{i}}-\mathrm{Xm}\right)^{2} .
$$

Nuevamente, aquí la sumatoria comprende de 1 a $N$. La variancia de la población es designada generalmente por $\sigma^{2}$. Los datos que poseen una variancia reducida están agrupados alrededor de su media, mientras que los datos con una variancia grande están dispersos con respecto a su media. La raíz cuadrada de la variancia es la desviación estándar $(S)$.

Entonces, si se desea estimar un parámetro estadístico poblacional por ejemplo la media, y se dispone de varias series o secuencias de datos, cada una con una media, tales medias forman otra serie a partir de la cual es posible calcular la variancia de la media. Un valor reducido de esta variancia significa una estimación más confiable de la media, ya que implica menor dispersión y por lo tanto mayor precisión (Fiering,1963).

\section{Regresión y correlación lineales}

Una relación lineal de la forma

$$
\mathrm{Y}=\alpha+\beta \cdot \mathrm{X}
$$

donde $Y$ y $X$ son los escurrimientos en dos corrientes, se denomina regresión lineal, y la técnica de mínimos cuadrados conduce a la expresión siguiente del coeficiente de regresión $\beta$ :

$$
\begin{gathered}
\beta=\operatorname{Cov}(\mathrm{X}, \mathrm{Y}) / \operatorname{Var}(\mathrm{X})= \\
\left(\Sigma \mathrm{X}_{\mathrm{i}} \mathrm{Y}_{\mathrm{i}}-\mathrm{N} \mathrm{Xm} \mathrm{Ym)} /\left(\Sigma \mathrm{X}_{\mathrm{i}}{ }^{2}-\mathrm{N} \mathrm{Xm}{ }^{2}\right)\right.
\end{gathered}
$$

La técnica de mínimos cuadrados también define al coeficiente de correlación $\left(r_{x y}\right)$ como una medida de la calidad o bondad del ajuste (dependencia) entre las variables $X$ y $Y$, estando definido como

$$
\begin{gathered}
r_{x y}=\operatorname{Cov}(X, Y) /[\operatorname{Var}(X) \operatorname{Var}(Y)]^{1 / 2} \\
r_{x y}=\left(\Sigma X_{i} Y_{i}-N X_{m} Y m\right) / \\
{\left[\left(\Sigma X_{i}^{2}-N X^{2}\right) 1 / 2\left(\Sigma Y_{i}^{2}-N m^{2}\right)^{1 / 2}\right]}
\end{gathered}
$$

Un valor de $r_{x y}$ igual a \pm 1 indica que las variables están perfectamente correlacionadas; en cambio, un valor de cero significa que no existe relación lineal entre ellas; los - valores intermedios definen el grado de ajuste o dependencia entre $X$ y $Y$. El coeficiente de correlación poblacional se designa por $\rho$. Entonces, la regresión corresponde a la ecuación matemática que relaciona las variables y la correlación, a la magnitud que indica el grado de asociación o dependencia entre las variables dependiente y la(s) independiente(s).

Hipótesis implícitas en la aplicación de la regresión

En lo que se denominará el modelo bidimensional, $X_{i}$ y $Y_{i}$ son los escurrimientos anuales en dos corrientes cercanas $(X, Y)$ o en dos estaciones hidrométricas próximas sobre el mismo río, con el arreglo siguiente en cuanto al tiempo (Fiering, 1963):

$$
\begin{aligned}
& X_{1}, X_{2}, \ldots X_{n 1}, \ldots X_{n 1+n 2} \\
& Y_{1}, Y_{2}, \ldots . Y_{n 1},
\end{aligned}
$$

donde $n l$ es el número de años del registro disponible en la estación $Y$, que además es común con la estación $X$; en esta última existen $n 2$ datos o términos adicionales que pueden 
ser utilizados para ampliar el registro $Y$ de acuerdo con la siguiente ecuación de la línea de tendencia:

$$
Y_{j}=Y m+\beta\left(X_{j}-X m\right)
$$

donde $j=1,2, \ldots, n 2$ y $Y m$ es la media de $\operatorname{los} n 1$ datos $Y_{i}$. La aplicación de la ecuación anterior implica las siguientes hipótesis:

Primera. Un régimen hidrológico estable existe, de manera que una correlación entre $X_{i}$ y $Y_{i}$, puede ser esperada.

Segunda. Los escurrimientos anuales, o alguna transformación de ellos, están distribuidos normalmente.

Tercera. La correlación serial de los escurrimientos anuales es cero.

¿Cuándo se debe usar la regresión?

Ya que los datos o una transformación de ellos están distribuidos normalmente, los primeros dos momentos estadísticos permiten especificar completamente su distribución. Entonces, un tópico importante será decidir cuándo los datos comunes y adicionales disponibles en la estación $X$ pueden ser utilizados para alcanzar una mejor estimación de los parámetros poblacionales del registro $Y$.

En virtud de que la magnitud de la variancia de cada estimación es una medida de la precisión asociada a ésta, entonces, si la variancia de un parámetro calculada utilizando los $n 1$ datos originales excede a la estimada empleando también los $n 2$ adicionales obtenidos por regresión (según la ecuación 6), está claro que el uso de la correlación mejora la estimación del parámetro. En cambio, si la variancia de un parámetro calculada con el registro ampliado excede a la estimada con el registro original, entonces, el registro combinado proporciona una estimación menos precisa del parámetro.

Se puede definir, para un arreglo específico A.1 y un parámetro estadístico en particular, con base en los valores de $n l$, $n 2$ y $\rho$, el Cociente de Información Relativa (CIR) como la relación entre la variancia de un parámetro, estimada a partir del registro original y la estimada con el registro ampliado. Cuando el CIR excede la unidad, una estimación más precisa se logra con el registro ampliado. Por lo tanto, la regresión no debe ser utilizada para ampliar el registro original cuando el CIR es menor que uno (Fiering,1963).

\section{Modelo bidimensional}

Cociente de información relativa de la media

Para un arreglo como A. 1 con datos $n 1, n 2$ y $r_{x y}$, se tiene que (Fiering, 1963)

$$
\begin{aligned}
& \mathrm{CIRm}=\{1-[\mathrm{n} 2 /(\mathrm{n} 1+\mathrm{n} 2)] \\
& \left.\left[\left(\mathrm{r}_{\mathrm{xy}}{ }^{2}(\mathrm{n} 1-2)-1\right) /(\mathrm{n} 1-3)\right]\right\}^{-1} .
\end{aligned}
$$

Resolviendo la ecuación anterior para $C I R m=1.0$, se obtiene el valor crítico

por lo cual:

$$
\mathrm{r}_{\mathrm{xy}}=[1 /(\mathrm{n} 1-2)]^{1 / 2}
$$

\begin{tabular}{ccccccccccc}
\hline $\mathrm{n} 1$ & 5 & 10 & 15 & 20 & 25 & 30 & 40 & 50 & 75 & 100 \\
\hline \hline $\mathrm{r}_{\mathrm{xy}}$ & 0.577 & 0.354 & 0.277 & 0.236 & 0.209 & 0.189 & 0.162 & 0.144 & 0.117 & 0.101 \\
\hline \hline
\end{tabular}

Cociente de información relativa de la variancia

Para un arreglo como el A. 1 con datos $n 1, n 2$ y $r_{x y}$, se tiene que (Fiering,1963)

$$
\mathrm{CIRv}=[2 /(\mathrm{n} 1-1)] / \operatorname{EMC}[\operatorname{Var}(\mathrm{Y})],
$$

siendo $E M C$ el error medio cuadrático de la variancia de las $Y$ :

$$
\begin{gathered}
\mathrm{EMC}[\operatorname{Var}(\mathrm{Y})]=[2 /(\mathrm{n} 1-1)] \\
+\mathrm{D}[2 \mathrm{~A}+(\mathrm{n} 2+2) \mathrm{B}+(\mathrm{n} 1+\mathrm{n} 2-1) \mathrm{C}-\mathrm{F}]
\end{gathered}
$$

donde:

$$
\begin{aligned}
\mathrm{A}= & (\mathrm{n} 1-1) \mathrm{r}_{\mathrm{xy}}{ }^{4}+(\mathrm{n} 1+4) \mathrm{r}_{\mathrm{xy}}{ }^{2}\left(1-\mathrm{r}_{\mathrm{xy}}{ }^{2}\right)+\left[(\mathrm{n} 1+1)\left(1-\mathrm{r}_{\mathrm{xy}}{ }^{2}\right) 2\right. \\
& /(\mathrm{n} 1-3)] \\
\mathrm{B}= & \mathrm{r}_{\mathrm{xy}}{ }^{4}+\left[6 \mathrm{r}_{\mathrm{xy}}{ }^{2}\left(1-\mathrm{r}_{\mathrm{xy}}{ }^{2}\right) /(\mathrm{n} 1-3)\right]+\left\{3\left(1-\mathrm{r}_{\mathrm{xy}}{ }^{2}\right) /\right. \\
& {[(\mathrm{n} 1-3)(\mathrm{n} 1-5)]\} } \\
\mathrm{C}= & {\left[2(\mathrm{n} 1-4)\left(1-\mathrm{r}_{\mathrm{xy}}{ }^{2}\right)\right] /(\mathrm{n} 1-3) } \\
\mathrm{D}= & \mathrm{n} 2 /(\mathrm{n} 1+\mathrm{n} 2-1)^{2} \\
\mathrm{~F}= & {[(\mathrm{n} 1+1)(2 \mathrm{n} 1+\mathrm{n} 2-2)] /(\mathrm{n} 1-1) }
\end{aligned}
$$

Para los valores típicos o de la práctica de $n 1$ y $n 2, C I R v$ excede la unidad cuando $r_{x y}$ es igual o mayor de 0.80 , de donde se deduce que las condiciones deseables que mejoran las estimaciones de la variancia son mucho más restrictivas que aquéllas que mejoran las estimaciones de la media (ecuación 8).

\section{Modelo tridimensional}

Arreglo y ecuación de regresión

En este caso, las estimaciones de media y variancia en un sitio $Z$ pueden ser mejoradas por correlación con base en los escurrimientos anuales en dos sitios cercanos $X$ y $Y$, cuyo arreglo en el tiempo es:

$$
\begin{aligned}
& X_{1}, X_{2}, \ldots \ldots X_{n 1}, \ldots . X_{n 1+n 2} \\
& Y_{1}, Y_{2}, \ldots \ldots Y_{n 1}, \ldots \ldots Y_{n 1+n 2} \\
& Z_{1}, Z_{2}, \ldots \ldots Z_{n 1}
\end{aligned}
$$


La ecuación de regresión trivariada utilizada para ampliar los datos es (Fiering,1963)

$$
\mathrm{Z}_{\mathrm{j}}=\mathrm{Zm}+\mathrm{bx}\left(\mathrm{X}_{\mathrm{j}}-\mathrm{Xm}\right)+\text { by }\left(\mathrm{Y}_{\mathrm{j}}-\mathrm{Ym}\right)
$$

en la cual, $j$ varía de 1 a $n 2, Z m$ es la media de los datos $Z j, b x$ y by son los coeficientes parciales de regresión calculados a partir de los $n 1$ datos comunes en las tres corrientes o los tres sitios; sus ecuaciones de acuerdo a la teoría de mínimos cuadrados son

$$
\begin{gathered}
\mathrm{bx}=[\operatorname{Var}(\mathrm{Y}) \cdot \operatorname{Cov}(\mathrm{X}, \mathrm{Z})-\operatorname{Cov}(\mathrm{X}, \mathrm{Y}) \cdot \operatorname{Cov}(\mathrm{Y}, \mathrm{Z})] / \Delta, \\
\mathrm{by}=[\operatorname{Var}(\mathrm{X}) \cdot \operatorname{Cov}(\mathrm{Y}, \mathrm{Z})-\operatorname{Cov}(\mathrm{X}, \mathrm{Y}) \cdot \operatorname{Cov}(\mathrm{X}, \mathrm{Z})] / \Delta, \\
\Delta=\operatorname{Var}(\mathrm{X}) \cdot \operatorname{Var}(\mathrm{Y})-[\operatorname{Cov}(\mathrm{X}, \mathrm{Y})]^{2} \cdot
\end{gathered}
$$

Cociente de información relativa de la media

Para un arreglo como el A.2 con datos $n 1$ y $n 2$, se tiene que (Fiering, 1963)

$\mathrm{CIRM}=\left\{1-(\mathrm{n} 2 / \mathrm{N})\left[\left(\mathrm{R}^{2}(\mathrm{n} 1-2)-2\right) /(\mathrm{n} 1-4)\right]\right\}^{-1}$,

siendo $N=n 1+n 2 . \mathrm{R}^{2}$ es el coeficiente de correlación total de $Z$ sobre $X$ y $Y$ :

$$
R^{2}=\left(r_{z x}^{2}+r_{z y}^{2}-2 r_{x y} r_{z x} r_{z y}\right) /\left(1-r_{x y}^{2}\right)
$$

Cociente de información relativa de la variancia

Para un arreglo como el A.2 con datos $n 1$ y $n 2$, se tiene que (Fiering, 1963)

$$
\mathrm{CIRV}=2 /\left[(\mathrm{n} 1-1) \operatorname{EMC}\left(\mathrm{S}^{2}\right)\right],
$$

en la cual

$$
\begin{gathered}
\mathrm{EMC}\left(\mathrm{S}^{2}\right)=\left[1 /(\mathrm{N}-1)^{2}\right]\{2(\mathrm{~N}-1)+4 \mathrm{n} 2 \mathrm{G} \\
+\mathrm{n} 2(\mathrm{n} 2-6) \mathrm{G}^{2}+4 \mathrm{n} 2(\mathrm{n} 2+3) \mathrm{G} /(\mathrm{n} 1-4)-4 \mathrm{n} 2 \\
\left.(2 \mathrm{n} 2+1) \mathrm{G}^{2} /(\mathrm{n} 1-4)+8 \mathrm{n} 2(\mathrm{n} 2+2) \mathrm{G}^{2} /[(\mathrm{n} 1-4)(\mathrm{n} 1-6)]\right\}
\end{gathered}
$$

siendo:

$$
G=\left(1-R^{2}\right)
$$

Restricciones inherentes de las ecuaciones del CIR

Las ecuaciones del $C I R$ de la variancia definen el número mínimo de $n l$ que en el caso del modelo bidimensional es cinco según la ecuación 10 (último denominador del parámetro $B$ ), y en el del modelo tridimensional es seis como se deduce del último denominador de la expresión del $E M C\left(S^{2}\right)$.

La comparación entre los valores de las expresiones del $C I R v$ y $C I R V$ conduce al hecho de que raramente la información estadística ganada en el modelo tridimensional excede, y generalmente es menor que la ganancia asociada con el modelo bidimensional (Fiering,1963).

Restricciones relativas a la homogeneidad hidrometeorológica

En el modelo bidimensional y principalmente en el trivariado es conveniente asegurarse de que las cuencas de los registros hidrométricos utilizados, formen parte de una región homogénea desde un punto de vista hidrometorológico, con lo cual se garantice de alguna manera su comportamiento similar y se acepte por lo tanto una relación causa-efecto semejante en todos los registros utilizados (Campos, 1991).

\section{Aplicaciones numéricas}

Modelo bidimensional en el río Piaxtla

Información disponible. La cuenca total del río Piaxtla es de aproximadamente $7222 \mathrm{~km}^{2}$ y su parteaguas define la mayor porción de la frontera sur de la región hidrológica No. 10 (Sinaloa), junto con la cuenca del río Quelite. La estación hidrométrica Piaxtla, operada por la Comisión Federal de Electricidad, tiene una cuenca de $5307 \mathrm{~km}^{2} \mathrm{e}$ inició su operación en octubre de 1957. La estación de aforos Ixpalino drena $6166 \mathrm{~km}^{2}$, ésta pertenecía a la Secretaría de Recursos Hidráulicos e inició su operación en octubre de 1952 (SRH,1970). La información disponible sobre volúmenes escurridos anuales en $\mathrm{Mm}^{3}$ en las estaciones Piaxtla e Ixpalino, ambas en el río Piaxtla, ha sido tomada de la lámina II-04 (SRH, 1975) y abarca desde el comienzo de la operación hasta 1973, con el año 1961 incompleto para la estación Piaxtla; tal información se tiene en la tabla 1 . 
Tabla 1. Información hidrométrica en las estaciones del río Piaxtla que se indican. Sinaloa

\begin{tabular}{|c|c|c|c|c|c|}
\hline \multirow[t]{2}{*}{ Año } & \multicolumn{2}{|c|}{ Vol. esc.anual $\left(\mathrm{Mm}^{3}\right)$} & \multirow[t]{2}{*}{ Año } & \multicolumn{2}{|c|}{ Vol. esc.anual $\left(\mathrm{Mm}^{3}\right)$} \\
\hline & Ixpalino & Piaxtla & & Ixpalino & Piaxtla \\
\hline 1973 & 1953.6 & 1122.4 & 1962 & 948.8 & 803.1 \\
\hline 1972 & 1678.4 & 980.4 & 1961 & 2208.2 & $1570.7+$ \\
\hline 1971 & 1413.7 & 829.8 & 1960 & 826.8 & 673.9 \\
\hline 1970 & 1699.5 & 1081.3 & 1959 & 975.1 & 737.7 \\
\hline 1969 & 1101.6 & 800.7 & 1958 & 1943.1 & 1501.4 \\
\hline 1968 & 2754.4 & 2364.9 & 1957 & 331.4 & $145.8^{*}$ \\
\hline 1967 & 1598.1 & 1133.6 & 1956 & 632.1 & $381.9^{*}$ \\
\hline 1966 & 1906.7 & 1360.9 & 1955 & 1668.6 & $1195.5^{*}$ \\
\hline 1965 & 816.0 & 506.7 & 1954 & 1145.7 & $785.0^{*}$ \\
\hline 1964 & 1472.2 & 988.3 & 1953 & 951.0 & $632.2 *$ \\
\hline 1963 & 1783.4 & 1402.3 & & & \\
\hline
\end{tabular}

+ Valor completado, ver inciso siguiente

* valores estimados, ver inciso: estimación de datos adicionales

Deducción de datos faltantes. Al revisar el año de 1961 en la estación Piaxtla, con base en el resumen mensual (SRH,1975), se observó que solo existía un mes faltante, junio. Por lo anterior, el planteamiento para estimar el volumen escurrido de ese mes fue realizar un análisis probabilístico con base en los 15 datos disponibles, a través del ajuste de una distribución Pearson tipo III
(Campos,1988) para obtener la mediana, la moda y el escurrimiento con $75 \%$ de probabilidad de excedencia; los resultados fueron: $14.504,8.005$ y $8.268 \mathrm{Mm}^{3}$, respectivamente. Se adoptó el valor de la moda y, entonces, el volumen escurrido anual del año 1961 se estimó en $1570.652 \mathrm{Mm}^{3}$. Los datos disponibles en junio se presentan en la tabla 2.

Tabla 2. Volúmenes escurridos en junio en la estación hidrométrica Piaxtla

\begin{tabular}{rr|rr|rr}
\hline Año & Vol. esc. & Año & Vol. esc. & Año & Vol. esc. \\
\hline \hline 1958 & 77.103 & 1964 & 22.288 & 1969 & 7.641 \\
1959 & 13.824 & 1965 & 4.202 & 1970 & 15.021 \\
1960 & 4.190 & 1966 & 12.684 & 1971 & 32.119 \\
1962 & 9.035 & 1967 & 13.097 & 1972 & 15.059 \\
1963 & 17.179 & 1968 & 9.894 & 1973 & 8.769 \\
\hline
\end{tabular}

Verificación de las hipótesis implícitas en la regresión. El empleo de la ecuación $5 \mathrm{~b}$ en los 16 datos comunes de las estaciones Ixpalino $(X)$ y Piaxtla $(Y)$ condujo al valor $r_{x y}=$ 0.9377 , por lo cual, la estadística $z$ (apéndice) toma un valor de 6.1967 , el cual es mayor que el valor crítico de 1.645 y por lo tanto el $r_{x y}$ calculado es significativo. Por otra parte, el valor crítico $r_{o}$ para 14 grados de libertad resulta de 0.497 , el cual es menor que el $r_{x y}$ calculado; entonces éste es significativo. De acuerdo con el procedimiento expuesto en el apéndice se obtuvieron los siguientes resultados:

\begin{tabular}{cccccc}
\hline \hline Estación & Valor W calculado & W crítico & Resultado & Cs & K \\
\hline Ixpalino $(n=21)$ & 0.977 & 0.908 & normal & 0.228 \\
Piaxtla $(n=16)$ & 0.900 & 0.887 & normal & 1.378 & 6.093 \\
\hline
\end{tabular}


donde $n=n 1+n 2$. Cs y $K$ son los coeficientes de asimetría y curtosis, cuyos valores para la distribución Normal son 0.000 y 3.000 , respectivamente; por lo tanto, se deduce que los datos muestrales de la estación hidrométrica Ixpalino son mucho más normales que los de la estación Piaxtla. Sin embargo, ambos registros cumplen con la condición de normalidad de acuerdo al test $W$. Por otra parte, el empleo de las ecuaciones I.5 y I.6 del apéndice condujo a los siguientes resultados:

\begin{tabular}{cccc}
\hline \hline Estación & $r_{l}$ calculado & $r_{l}$ crítico & Resultado \\
\hline \hline Ixpalino $(\mathrm{n}=21)$ & -0.177 & -0.477 & independiente \\
Piaxtla $(\mathrm{n}=16)$ & -0.276 & -0.556 & independiente
\end{tabular}

Por lo tanto, ambas muestras cumplen con la condición de independencia.

Cálculo de los cocientes de información relativa. El orden cronológico inverso en el que se ha expuesto la información disponible en la tabla 1 obedece al planteamiento del modelo bidimensional o arreglo A.1, por lo cual, $n 1$ y $n 2$ toman valores de 16 y 5 , respectivamente. Para el valor calculado de $r_{x y}=0.9377$, la aplicación de las ecuaciones 7 y 9 condujo a los valores siguientes, para los cocientes de información relativa de la media y de la variancia:

$$
C I R m=1.261 ; C I R v=1.225,
$$

de donde se deduce que la ampliación del registro de la estación hidrométrica Piaxtla, con base en los datos adicionales de la estación Ixpalino mejora la estimación de la media y la variancia.

Estimación de datos adicionales. Al aplicar la ecuación 4 se obtuvo un coeficiente de regresión de 0.785 ; el empleo de la ecuación 6 condujo a los valores presentados y destacados en la tabla 1 con un asterisco.

\section{Modelo tridimensional en el río Fuerte}

Información disponible. La cuenca del río Fuerte está localizada al norte de la región hidrológica No. 10. Sinaloa, su parteaguas define la frontera de esta región, es la más importante tanto por su extensión $\left(33590 \mathrm{~km}^{2}\right.$ hasta la estación hidrométrica San Blas), como por sus escurrimientos y la magnitud de las obras que en ella se han construido; abarca cuatro estados con los siguientes porcentajes: Chihuahua, 73.2; Sinaloa, 17.5; Sonora, 7.7; y Durango 1.6 (SRH,1970).

Las estaciones hidrométricas del río Fuerte utilizadas para ejemplificar el uso del modelo trivariado (arreglo A.2) fueron: Huites $(X)$ en el río Fuerte cuya área de cuenca es de $26020 \mathrm{~km}^{2}$, San Francisco ( $Y$ ) sobre el río San Miguel con un área drenada de $17531 \mathrm{~km}^{2}$ y Palo dulce $(Z)$ en el río Chínipas con $6323 \mathrm{~km}^{2}$. La disponibilidad de datos de volúmenes escurridos anuales de acuerdo al concentrado de tal información en la lámina II-04 (SRH,1975) es la siguiente: las estaciones Huites y San Francisco tienen datos en el periodo 1942 a 1973, con los años 1943 y 1944 incompletos para San Francisco; la estación Palo Dulce presenta información desde 1958 hasta 1973, con el año 1961 faltante.

Deducción de datos faltantes. Al revisar la información disponible en la estación hidrométrica Palo Dulce er el sistema BANDAS (CNA, 1997), se dedujo que existe información mensual en el periodo septiembre de 1957 a diciembre de 1976, con únicamente los meses de junio a noviembre faltantes en el año 1961. Por lo anterior, el planteamiento para la deducción de tales valores faltantes fue el análisis probabilístico por meses, con base en la distribución Pearson tipo III (Campos, 1988) para estimar la moda, y cuando ésta no existe porque los datos definen una función de densidad tipo $J$ invertida, se estimó el valor cuya probabilidad de excedencia es de $75 \%$.

Bajo tal planteamiento, los valores estimados para los meses de junio, octubre y noviembre correspondieron al escurrimiento cuya probabilidad de no excedencia es de $25 \%$, con los valores siguientes: $3.869,24.271$ y 7.746 $\mathrm{Mm}^{3}$, respectivamente. En el resto de los meses se empleó la moda con valores para julio, agosto y septiembre de: $109.087,192.598$ y $140.861 \mathrm{Mm}^{3}$, respectivamente. Con base en tales estimaciones y los datos disponibles, el escurrimiento anual de 1961 se estimó en $797.204 \mathrm{Mm}^{3}$. Como ejemplo de éstos análisis, en la tabla 3 se presentan los datos y resultados de los meses de junio y julio.

Tabla 3. Datos y resultados probalísticos en la estación Palo Dulce

\begin{tabular}{|c|c|c|c|c|c|}
\hline Año & Vol. esc. $\left(\mathrm{Mm}^{3}\right)$ & Vol. esc. $\left(\mathrm{Mm}^{3}\right)$ & Año & Vol. esc. $\left(\mathrm{Mm}^{3}\right)$ & Vol. esc. $\left(\mathrm{Mm}^{3}\right)$ \\
\hline & junio & julio & & junio & julio \\
\hline 1958 & 42.868 & 125.844 & 1963 & 2.879 & 94.279 \\
\hline 1959 & 8.634 & 137.007 & 1964 & 4.561 & 122.452 \\
\hline 1960 & 4.677 & 75.373 & 1965 & 1.546 & 93.538 \\
\hline 1962 & 4.771 & 165.946 & 1966 & 26.512 & 237.343 \\
\hline
\end{tabular}


Tabla 3. Datos y resultados probalísticos en la estación Palo Dulce (continuación)

\begin{tabular}{rcc|ccc}
\hline Año & Vol esc. $\left(\mathrm{Mm}^{3}\right)$ & Vol esc. $\left(\mathrm{Mm}^{3}\right)$ & Año & Vol esc. $\left(\mathrm{Mm}^{3}\right)$ & Vol esc. $\left(\mathrm{Mm}^{3}\right)$ \\
\hline \hline & junio & julio & & junio & julio \\
\hline 1967 & 31.727 & 198.649 & 1974 & 4.438 & 194.481 \\
1968 & 10.883 & 306.711 & 1975 & 3.318 & 155.158 \\
1969 & 1.869 & 364.868 & 1976 & 10.271 & 192.623 \\
1970 & 1.507 & 117.701 & Media & 15.265 & 171.938 \\
1971 & 62.905 & 270.443 & Mediana $[\mathrm{P}(\mathrm{X}<\mathrm{x})=0.50]$ & 10.030 & 160.511 \\
1972 & 47.382 & 178.784 & Moda & $\ldots$ & 136.869 \\
1973 & 4.025 & 63.680 & Esc. con $\mathrm{P}(\mathrm{X}<\mathrm{x})=0.25$ & 3.869 & 116.703 \\
\hline \hline
\end{tabular}

Planteamiento de los análisis. Nuevamente se utilizarán los datos disponibles en un orden cronológico inverso para cumplir con el arreglo A.2, de manera que ahora $n 1$ y $n 2$ serán igual a 12 (1973 -1962) y cuatro (1961-1958), respectivamente. Esta concepción en la aplicación del modelo tridimensional a la estación Palo Dulce permite tener un contraste de los cuatro valores estimados contra volúmenes anuales reales, uno de ellos, el del año 1961 estimado. Los volúmenes escurridos anuales $\left(\mathrm{Mm}^{3}\right)$ a utilizar son los mostrados en la tabla 4:

Tabla 4. Información hidrométrica en las estaciones que se indican del río Fuerte. Sinaloa

\begin{tabular}{cccc}
\hline \hline Año & Vol. esc. (X) & Vol. esc. (Y) & Vol. esc. (Z) \\
\hline 1973 & Huites & San Francisco & Palo Dulce \\
\hline 1972 & 6117.1 & 3837.9 & 954.6 \\
1971 & 5546.7 & 3566.3 & 1044.4 \\
1970 & 4494.1 & 2138.6 & 1477.4 \\
1969 & 3252.5 & 2083.7 & 632.8 \\
1968 & 2599.3 & 1432.4 & 799.2 \\
1967 & 6725.6 & 4238.1 & 1205.8 \\
1966 & 3968.7 & 2470.9 & 946.2 \\
1965 & 5991.9 & 3764.3 & 1330.5 \\
1964 & 2826.3 & 1561.3 & 727.2 \\
1963 & 3326.1 & 2239.6 & 677.5 \\
1962 & 4220.1 & 2891.2 & 649.8 \\
1961 & 2679.5 & 1992.3 & 637.9 \\
1960 & 4414.1 & 3123.9 & $825.0^{*}$ \\
1959 & 5615.1 & 3862.1 & $1575.3 *$ \\
1958 & 3559.7 & 2633.0 & $1089.3 *$ \\
\hline
\end{tabular}


Verificación de las hipótesis implícitas en la regresión. $\mathrm{El}$ empleo de la ecuación 5 b en los 12 datos comunes de las estaciones Huites $(X)$, San Francisco $(Y)$ y Palo Dulce $(Z)$ condujo a los valores: $r_{x y}=0.962, r_{z x}=0.689$ y $r_{z y}=0.500$, por lo cual la estadística $z$ (apéndice) toma un valor de 1.648 en el caso más desfavorable, el cual es escasamente mayor que el valor crítico de $1.645 \mathrm{y}$, por lo tanto, $r_{z y}$ es aún significativo. Por otra parte, el valor crítico $r_{o}$ para 10 grados de libertad resulta ser 0.576 , el cual es mayor que el $r_{z y}$ calculado, entonces éste no es diferente de cero.

Con base en el procedimiento expuesto en el apéndice se obtuvieron los siguientes resultados:

\begin{tabular}{cccccc}
\hline \hline Estación & W calculado & W crítico & Resultado & Cs & K \\
\hline \hline Huites $(n=16)$ & 0.938 & 0.887 & normal & 0.368 & 2.518 \\
San Fco. $(n=16)$ & 0.937 & 0.887 & normal & -0.013 & 2.201 \\
Palo Dulce $(n=12)$ & 0.890 & 0.859 & normal & 0.756 & 3.153 \\
\hline
\end{tabular}

Nuevamente, $n=n 1+n 2$. Todos los registros cumplen con la condición de normalidad y sus coeficientes de asimetría y curtosis se aproximan a los de la distribución Normal, cero y tres, respectivamente. El empleo de las ecuaciones I. 5 y I.6 del apéndice condujo a los resultados siguientes:

\begin{tabular}{cccc}
\hline \hline Estación & $r_{l}$ calculado & $r_{l}$ crítico & Resultado \\
\hline Huites $(\mathrm{n}=16)$ & -0.130 & -0.556 & independiente \\
San Fco. $(\mathrm{n}=16)$ & -0.168 & -0.556 & independiente \\
Palo Dulce $(\mathrm{n}=12)$ & 0.047 & 0.382 & independiente \\
\hline \hline
\end{tabular}

Por lo tanto, los tres registros cumplen con la condición de independencia.

Cálculo de los cocientes de información relativa. Con base en los valores de $r_{x y}, r_{z x}$ y $r_{z y}$ la aplicación de la ecuación 15 aporta un valor de 0.915 para el coeficiente de correlación total y las ecuaciones 14 y 16 conducen a los valores siguientes, para los cocientes de información relativa de la media y variancia:

$$
C I R M=1.249 ; C I R V=1.193,
$$

de donde se deduce que la ampliación del registro de la estación hidrométrica Palo Dulce, con base en los datos de las estaciones Huites y San Francisco, mejora sensiblemente la estimación de la media y la variancia.

Deducción de datos adicionales. Al aplicar las ecuaciones 12 y 13 se obtuvieron los valores siguientes 0.5601 y -0.6763 para los coeficientes parciales de regresión. Por otra parte, con el uso de la ecuación 11 se obtienen las estimaciones de los valores de $Z_{j}$ que se deducen; éstos fueron: $683.6,857.0,537.1$ y $199.1 \mathrm{Mm}^{3}$.
Al comparar los valores anuales anteriores contra los volúmenes escurridos reales, la similitud es escasa; por ello, se realizaron los siguientes tres planteamientos y sus respectivos análisis numéricos:

1) Aplicar las ecuaciones 12,13 y 11 a los logaritmos naturales de los datos $X_{i}, Y_{i}$ y $Z_{i}$, lo cual implica realizar una regresión múltiple exponencial del tipo: $Z_{j}=a X^{b} Y^{c}$ (Campos, 1992a).

2) Aplicar el modelo bidimensional entre las estaciones Huites $(X)$ y Palo Dulce $(Y)$; los resultados fueron $r_{x y}=$ $0.689, \beta=0.137, C I R m=1.116$ y $C I R v=1.050$.

3) Aplicar el modelo bidimensional entre las estaciones San Francisco $(X)$ y Palo Dulce $(Y)$; los resultados fueron $r_{x y}=0.500, \beta=0.152, C I R m=1.043$ y $C I R v=1.039$.

En la tabla 5 se contrastan los volúmenes escurridos anuales (VEA) reales, de la estación Palo Dulce en el periodo 1961-1958, contra los estimados con el modelo tridimensional y los obtenidos con cada uno de los tres planteamientos anteriores: 
Tabla 5. Contraste de resultados en la estación hidrométrica Palo Dulce

\begin{tabular}{|c|c|c|c|c|}
\hline VEA real $(\mathrm{X})$ & VEA reg. lineal (Y1) & VEA reg. exponencial (Y2) & Mod. bidim. con Huites (Y3) & Mod. bidim. con San Fco. (Y4) \\
\hline 825.0 & 683.6 & 771.8 & 937.5 & 990.5 \\
\hline 1575.3 & 857.0 & 937.3 & 1101.6 & 1103.0 \\
\hline 1089.3 & 537.1 & 632.2 & 820.8 & 915.7 \\
\hline \multirow[t]{2}{*}{999.6} & 199.1 & 559.4 & 908.3 & 1072.7 \\
\hline & $r_{x y 1}=0.531$ & $\mathrm{r}_{\mathrm{xy2}}=0.658$ & $r_{x y 3}=0.723$ & $\mathrm{r}_{\mathrm{xy} 4}=0.532$ \\
\hline
\end{tabular}

\section{Conclusiones}

Primera. En este trabajo se han presentado las ecuaciones relativas a los modelos bi y tridimensionales de ampliación de registros de volúmenes escurridos anuales, haciendo uso de la correlación y la regresión lineal cuando ello es conveniente desde un punto de vista estadístico. Además, se han expuesto las herramientas estadísticas adicionales (apéndice) que son necesarias para la aplicación a casos reales.

Segunda. Se ha expuesto toda la información hidrométrica utilizada en las aplicaciones numéricas descritas (tablas 1 y 4) y se han detallado y comentado los resultados parciales y finales, incluyendo sus análisis complementarios (tabla 5) que se estimaron necesarios para llegar a resultados más confiables (caso trivariado).

Tercera. Debido a la complejidad numérica de algunas de las ecuaciones; por ejemplo, las asociadas al cociente de información relativa de la variancia, de ambos modelos (CIRv y CIRV), así como las correspondientes a las ecuaciones de regresión, se desarrollaron programas de cómputo en Basic que las resuelven, los cuales están disponibles con el autor.

\section{Apéndice. Pruebas para las hipótesis de existencia de correlación, normalidad e independencia}

Prueba para el coeficiente de correlación

Para probar si existe la posibilidad de que el coeficiente de correlación poblacional ( $\rho$ ) sea cero, aun cuando $r_{x y}, r_{z x}$ y/o $r_{z y}$ hayan resultado diferentes de cero, se evalúa la estadística

$$
z=\left[(\mathrm{n} 1-3)^{1 / 2} / 2\right] \operatorname{Ln}\left[\left(1+\mathrm{r}_{\mathrm{xy}}\right) /\left(1-\mathrm{r}_{\mathrm{xy}}\right)\right]
$$

la cual se compara con el valor $z c$ que tiene distribución normal para un cierto nivel de confianza, comúnmente $95 \%$, por lo cual, $z c=1.645$ (CFE,1970). Si $z>z c$ no hay posibilidad de que $\rho$ sea igual a cero y por lo tanto, $r_{x y}$ es significativo al 5\% de nivel de significancia (Campos,1992a).

Otro criterio consiste en comparar el valor calculado del coeficiente de correlación $\left(r_{x y}\right)$ contra el tabulado $\left(r_{o}\right)$ con $v=\mathrm{n} 1-2$ grados de libertad; si el primero excede al segundo no existe posibilidad de que $\rho$ sea igual a cero. Para un nivel de significancia de 5\%, Maisel (1973) presenta los siguientes valores de $r_{o}$ :

\begin{tabular}{rrrrrrrrr}
\hline$v$ & $\mathrm{r}_{0}$ & $v$ & $\mathrm{r}_{0}$ & $v$ & $\mathrm{r}_{0}$ & $v$ & $\mathrm{r}_{0}$ \\
\hline 8 & 0.632 & 15 & 0.482 & 22 & 0.404 & 29 & 0.355 \\
9 & 0.602 & 16 & 0.468 & 23 & 0.396 & 30 & 0.349 \\
10 & 0.576 & 17 & 0.456 & 24 & 0.388 & 35 & 0.325 \\
11 & 0.553 & 18 & 0.444 & 25 & 0.381 & 40 & 0.304 \\
12 & 0.532 & 19 & 0.433 & 26 & 0.374 & 45 & 0.288 \\
13 & 0.514 & 20 & 0.423 & 27 & 0.367 & 50 & 0.273 \\
14 & 0.497 & 21 & 0.413 & 28 & 0.361 & 60 & 0.250 \\
\hline \hline
\end{tabular}


Prueba de normalidad

El test W de Shapiro \& Wilk permite verificar si es probable que una muestra provenga de una población normal; su procedimiento es el siguiente (Ruiz-Maya,1977): en la muestra de $n$ elementos $(n<50)$ denominados $x_{i}$, se ordenan éstos de menor a mayor, siendo ahora el i-ésimo elemento $\mathrm{X}_{i}$. En seguida se calculan las cantidades siguientes:

$$
S^{2}=\Sigma_{1}\left(X_{i}-X m\right)^{2}
$$

$$
\begin{gathered}
b=\Sigma_{2} a_{n, k}\left(X_{n-i+1}-X_{i}\right) \\
W=b^{2} / S^{2}
\end{gathered}
$$

La sumatoria 1 abarca de $\mathrm{i}=1$ a $\mathrm{i}=\mathrm{n}$ y la sumatoria 2 de $\mathrm{i}=1 \mathrm{a} \mathrm{i}=\mathrm{k}$. Si $n$ es par, entonces $k=n / 2$ y si $n$ es impar $k=$ $(n-1) / 2 ; a_{n, k}$ son los coeficientes que se tienen en la tabla I.1. Si el valor calculado de $W$ es mayor que el de la tabla I.2, se acepta la hipótesis que establece que la muestra proviene de una población normal en el nivel de significancia $(\alpha)$ adoptado, comúnmente 5\% (Ruiz-Maya, 1977;

\begin{tabular}{|c|c|c|c|c|c|c|c|c|c|c|c|}
\hline $\mathrm{k} / \mathrm{n}^{\mathrm{n}}$ & 10 & 11 & 12 & 13 & 14 & 15 & 16 & 17 & 18 & 19 & 20 \\
\hline 1 & 0.5739 & 0.5601 & 0.5475 & 0.5359 & 0.5251 & 0.5150 & 0.5056 & 0.4968 & 0.4886 & 0.4808 & 0.4734 \\
\hline 2 & .3291 & .3315 & .3325 & .3325 & .3318 & .3306 & .3290 & .3273 & .3253 & .3232 & .3211 \\
\hline 3 & .2141 & .2260 & .2347 & .2412 & .2460 & .2495 & .2521 & .2540 & .2553 & .2561 & .2565 \\
\hline 4 & .1224 & .1429 & .1586 & .1707 & .1802 & .1878 & .1939 & .1988 & .2027 & .2059 & .2085 \\
\hline 5 & .0399 & .0695 & .0922 & .1099 & .1240 & .1353 & .1447 & .1524 & .1587 & .1641 & .1686 \\
\hline 6 & & 0.0000 & 0.0303 & 0.0539 & 0.0727 & 0.0880 & 0.1005 & 0.1109 & 0.1197 & 0.1271 & 0.1334 \\
\hline 7 & & . & . & .0000 & .0240 & .0433 & .0593 & .0725 & .0837 & .0932 & .1013 \\
\hline 8 & & . & . & . & . & .0000 & .0196 & .0359 & .0496 & .0612 & .0711 \\
\hline 9 & & - & - & - & . & . & - & .0000 & .0163 & .0303 & .0422 \\
\hline 10 & & - & - & . & . & - & . & . & . & .0000 & .0140 \\
\hline $\mathrm{k} / \mathrm{n}^{\mathrm{n}}$ & 21 & 22 & 23 & 24 & 25 & 26 & 27 & 28 & 29 & 30 & \\
\hline 1 & 0.4643 & 0.4590 & 0.4542 & 0.4493 & 0.4450 & 0.4407 & 0.4366 & 0.4328 & 0.4291 & 0.4254 & \\
\hline 2 & .3185 & .3156 & .3126 & .3098 & .3069 & .3043 & .3018 & .2992 & .2968 & .2944 & \\
\hline 3 & .2578 & .2571 & .2563 & .2554 & .2543 & .2533 & .2522 & .2510 & .2499 & .2487 & \\
\hline 4 & .2119 & .2131 & .2139 & .2145 & .2148 & .2151 & .2152 & .2151 & .2150 & .2148 & \\
\hline 5 & .1736 & .1764 & .1787 & .1807 & .1822 & .1836 & .1848 & .1857 & .1864 & .1870 & \\
\hline 6 & 0.1399 & 0.1443 & 0.1480 & 0.1512 & 0.1539 & 0.1563 & 0.1584 & 0.1601 & 0.1616 & 0.1630 & \\
\hline 7 & .1092 & .1150 & .1201 & .1245 & .1283 & .1316 & .1346 & .1372 & .1395 & .1415 & \\
\hline 8 & .0804 & .0878 & .0941 & .0997 & .1046 & .1089 & .1128 & .1162 & .1192 & .1219 & \\
\hline 9 & .0530 & .0618 & .0696 & .0764 & .0823 & .0876 & .0923 & .0965 & .1002 & .1036 & \\
\hline 10 & .0263 & .0368 & .0459 & .0539 & .0610 & .0672 & .0728 & .0778 & .0822 & .0862 & \\
\hline 11 & 0.0000 & 0.0122 & 0.0228 & 0.0321 & 0.0403 & 0.0476 & 0.0540 & 0.0598 & 0.0650 & 0.0697 & \\
\hline 12 & . & . & .0000 & .0107 & .0200 & .0284 & .0358 & .0424 & .0483 & .0537 & \\
\hline 13 & . & - & . & . & .0000 & .0094 & .0178 & .0253 & .0320 & .0381 & \\
\hline 14 & . & - & - & - & - & . & .0000 & .0084 & .0159 & .0227 & \\
\hline 15 & , & - & - & . & . & . & . & , & .0000 & .0076 & \\
\hline
\end{tabular}
Campos, 1992b).

Tabla I.1. Coeficientes $a_{n, k}$ del test W de Shapiro \& Wilk (Ruiz-Maya, 1977) 
Tabla I.1. Coeficientes $a_{n, k}$ del test W de Shapiro \& Wilk (Ruiz-Maya,1977) (continuación)

\begin{tabular}{|c|c|c|c|c|c|c|c|c|c|c|}
\hline $\mathrm{k} / \mathrm{n}^{\mathrm{n}}$ & 31 & 32 & 33 & 34 & 35 & 36 & 37 & 38 & 39 & 40 \\
\hline 1 & 0.4220 & 0.4188 & 0.4156 & 0.4127 & 0.4096 & 0.4068 & 0.4040 & 0.4015 & 0.3989 & 0.3064 \\
\hline 2 & .2921 & .2898 & .2876 & .2854 & .2834 & .2813 & .2794 & .2774 & .2755 & .2737 \\
\hline 3 & .2475 & .2463 & .2451 & .2439 & .2427 & .2415 & .2403 & .2391 & .2380 & .2368 \\
\hline 4 & .2145 & .2141 & .2137 & .2132 & .2127 & .2121 & .2116 & .2110 & .2104 & .2098 \\
\hline 5 & .1874 & .1878 & .1880 & .1882 & .1883 & .1883 & .1883 & .1881 & .1880 & .1878 \\
\hline 6 & 0.1641 & 0.1651 & 0.1660 & 0.1667 & 0.1673 & 0.1678 & 0.1683 & 0.1686 & 0.1689 & 0.1691 \\
\hline 7 & .1433 & .1449 & .1463 & .1475 & .1487 & .1496 & .1505 & .1513 & .1520 & .1526 \\
\hline 8 & .1243 & .1265 & .1284 & .1301 & .1317 & .1331 & .1344 & .1356 & .1366 & .1376 \\
\hline 9 & .1066 & .1093 & .1118 & .1140 & .1160 & .1179 & .1196 & .1211 & .1225 & .1237 \\
\hline 10 & .0899 & .0931 & .0961 & .0988 & .1013 & .1036 & .1056 & .1075 & .1092 & .1108 \\
\hline 11 & 0.0739 & 0.0777 & 0.0812 & 0.0844 & 0.0873 & 0.0900 & 0.0924 & 0.0947 & 0.0967 & 0.0986 \\
\hline 12 & .0585 & .0629 & .0669 & .0706 & .0739 & .0770 & .0798 & .0824 & .0848 & .0870 \\
\hline 13 & .0435 & .0485 & .0530 & .0572 & .0610 & .0645 & .0677 & .0706 & .0733 & .0759 \\
\hline 14 & .0289 & .0344 & .0395 & .0441 & .0484 & .0523 & .0559 & .0592 & .0622 & .0651 \\
\hline 15 & .0144 & .0206 & .0262 & .0314 & .0361 & .0404 & .0444 & .0481 & .0515 & .0546 \\
\hline 16 & 0.0000 & 0.0068 & 0.0131 & 0.0187 & 0.0239 & 0.0287 & 0.0331 & 0.0372 & 0.0409 & 0.0444 \\
\hline 17 & - & . & .0000 & .0062 & .0119 & .0172 & .0220 & .0264 & .0305 & .0343 \\
\hline 18 & - & - & - & . & .0000 & .0057 & .0110 & .0158 & .0203 & .0244 \\
\hline 19 & $\cdot$ & - & - & - & - & - & .0000 & .0053 & .0101 & .0146 \\
\hline 20 & - & . & - & . & - & - & - & - & .0000 & .0049 \\
\hline $\mathrm{k} / \mathrm{n}^{\mathrm{n}}$ & 41 & 42 & 43 & 44 & 45 & 46 & 47 & 48 & 49 & 50 \\
\hline 1 & 0.3940 & 0.3917 & 0.3894 & 0.3872 & 0.3850 & 0.3830 & 0.3808 & 0.3789 & 0.3770 & 0.3751 \\
\hline 2 & .2719 & .2701 & .2684 & .2667 & .2651 & .2635 & .2620 & .2604 & .2589 & .2574 \\
\hline 3 & .2357 & .2345 & .2334 & .2323 & .2313 & .2302 & .2291 & .2281 & .2271 & .2260 \\
\hline 4 & .2091 & .2085 & .2078 & .2072 & .2065 & .2058 & .2052 & .2045 & .2038 & .2032 \\
\hline 5 & .1876 & .1874 & .1871 & .1868 & .1865 & .1862 & .1859 & .1855 & .1851 & .1847 \\
\hline 6 & 0.1693 & 0.1694 & 0.1695 & 0.1695 & 0.1695 & 0.1695 & 0.1695 & 0.1693 & 0.1692 & 0.1691 \\
\hline 7 & .1531 & .1535 & .1539 & .1542 & .1545 & .1548 & .1550 & .1551 & .1553 & .1554 \\
\hline 8 & .1384 & .1392 & .1398 & .1405 & .1410 & .1415 & .1420 & .1423 & .1427 & .1430 \\
\hline 9 & .1249 & .1259 & .1269 & .1278 & .1286 & .1293 & .1300 & .1306 & .1312 & .1317 \\
\hline 10 & .1123 & .1136 & .1149 & .1160 & .1170 & .1180 & .1189 & .1197 & .1205 & .1212 \\
\hline 11 & 0.1004 & 0.1020 & 0.1035 & 0.1049 & 0.1062 & 0.1073 & 0.1085 & 0.1095 & 0.1105 & 0.1113 \\
\hline 12 & .0891 & .0909 & .0927 & .0943 & .0959 & .0972 & .0986 & .0998 & .1010 & 1020 \\
\hline 13 & .0782 & .0804 & .0824 & .0842 & .0860 & .0876 & .0892 & .0906 & .0919 & .0932 \\
\hline 14 & .0677 & .0701 & .0724 & .0745 & .0765 & .0783 & .0801 & .0817 & .0832 & .0846 \\
\hline
\end{tabular}


DOI: http://dx.doi.org/10.22201/fi.25940732e.1999.01n2.008

Tabla I.1. Coeficientes $a_{n, k}$ del test W de Shapiro \& Wilk (Ruiz-Maya,1977) (continuación)

\begin{tabular}{|c|c|c|c|c|c|c|c|c|c|c|}
\hline $\mathrm{k} /{ }^{\mathrm{n}}$ & 41 & 42 & 43 & 44 & 45 & 46 & 47 & 48 & 49 & 50 \\
\hline 15 & .0575 & .0602 & .0628 & .0651 & .0673 & .0694 & .0713 & .0731 & .0748 & .0764 \\
\hline 16 & 0.0476 & 0.0506 & 0.0534 & 0.0560 & 0.0584 & 0.0607 & 0.0628 & 0.0648 & 0.0667 & 0.0685 \\
\hline 17 & .0379 & .0411 & .0442 & .0471 & .0497 & .0522 & .0546 & .0568 & .0588 & .0608 \\
\hline 18 & .0283 & .0318 & .0352 & .0383 & .0412 & .0439 & .0465 & .0489 & .0511 & .0532 \\
\hline 19 & .0188 & .0227 & .0263 & .0296 & .0328 & .0357 & .0385 & .0411 & .0436 & .0459 \\
\hline 20 & .0094 & .0136 & .0175 & .0211 & .0245 & .0277 & .0307 & .0335 & .0361 & .0386 \\
\hline 21 & 0.0000 & 0.0045 & 0.0087 & 0.0126 & 0.0163 & 0.0197 & 0.0229 & 0.0259 & 0.0288 & 0.0314 \\
\hline 22 & - & . & .0000 & .0042 & .0081 & .0118 & .0153 & .0185 & .0215 & .0244 \\
\hline 23 & - & - & - & - & .0000 & .0039 & .0076 & .0111 & 0.143 & .0174 \\
\hline 24 & - & - & - & - & - & - & .0000 & .0037 & .0071 & .0104 \\
\hline 25 & . & . & - & - & . & . & . & . & .0000 & .0035 \\
\hline
\end{tabular}

Tabla I.2. Valores críticos de W en el test W de Shapiro \& Wilk (Ruiz-Maya, 1977)

\begin{tabular}{|c|c|c|c|c|c|c|c|c|c|}
\hline $\mathrm{n}$ & $\alpha=1 \%$ & $\alpha=2 \%$ & $\alpha=5 \%$ & $\alpha=10 \%$ & $\mathrm{n}$ & $\alpha=1 \%$ & $\alpha=2 \%$ & $\alpha=5 \%$ & $\alpha=10 \%$ \\
\hline 3 & 0.753 & 0.756 & 0.767 & 0.789 & 27 & .894 & .906 & .923 & .935 \\
\hline 4 & .687 & .707 & .748 & .792 & 28 & .896 & .908 & .924 & .936 \\
\hline 5 & .686 & .715 & .762 & .806 & 29 & .898 & .910 & .926 & .937 \\
\hline 6 & 0.713 & 0.743 & 0.788 & 0.826 & 30 & .900 & .912 & .927 & .939 \\
\hline 7 & .730 & .760 & .803 & .838 & 31 & 0.902 & 0.914 & 0.929 & 0.940 \\
\hline 8 & .749 & .778 & .818 & .851 & 32 & .904 & .915 & .930 & .941 \\
\hline 9 & .764 & .791 & .829 & .859 & 33 & .906 & .917 & .931 & .942 \\
\hline 10 & .781 & .806 & .842 & .869 & 34 & .908 & .919 & .933 & .943 \\
\hline 11 & 0.792 & 0.817 & 0.850 & 0.876 & 35 & .910 & .920 & .934 & .944 \\
\hline 12 & .805 & .828 & .859 & .883 & 36 & 0.912 & 0.922 & 0.935 & 0.945 \\
\hline 13 & .814 & .837 & .866 & .889 & 37 & .914 & .924 & .936 & .946 \\
\hline 14 & .825 & .846 & .874 & .895 & 38 & .916 & .925 & .938 & .947 \\
\hline 15 & .835 & .855 & .881 & .901 & 39 & .917 & .927 & .939 & .948 \\
\hline 16 & 0.844 & 0.863 & 0.887 & 0.906 & 40 & .919 & .928 & .940 & .949 \\
\hline 17 & .851 & .869 & .892 & .910 & 41 & 0.920 & 0.929 & 0.941 & 0.950 \\
\hline 18 & .858 & .874 & .897 & .914 & 42 & .922 & .930 & .942 & .951 \\
\hline 19 & .863 & .879 & .901 & .917 & 43 & .923 & .932 & .943 & .951 \\
\hline 20 & .868 & .884 & .905 & .920 & 44 & .924 & .933 & .944 & .952 \\
\hline 21 & 0.873 & 0.888 & 0.908 & 0.923 & 45 & .926 & .934 & .945 & .953 \\
\hline 22 & .878 & .892 & .911 & .926 & 46 & 0.927 & 0.935 & 0.945 & 0.953 \\
\hline 23 & .881 & .895 & .914 & .928 & 47 & .928 & .936 & .946 & .954 \\
\hline 24 & .884 & .898 & .916 & .930 & 48 & .929 & .937 & .947 & .954 \\
\hline
\end{tabular}


Tabla I.2. Valores críticos de W en el test W de Shapiro \& Wilk (Ruiz-Maya, 1977) (continuación)

\begin{tabular}{|c|c|c|c|c|c|c|c|c|c|}
\hline 25 & .888 & .901 & .918 & .931 & 49 & .929 & .937 & .947 & .955 \\
\hline 26 & 0.891 & 0.904 & 0.920 & 0.933 & 50 & .930 & .938 & .947 & .955 \\
\hline
\end{tabular}

Prueba de independencia

Se define como correlación serial o autocorrelación la dependencia existente entre un elemento de la muestra y otro distante $k$ elementos. Entonces, el coeficiente de correlación serial unitario estará definido por la expresión (WMO,1971)

$$
\begin{gathered}
\mathrm{r}_{1}=\left[(\mathrm{n}-1) \Sigma_{1} \mathrm{x}_{\mathrm{i}} \cdot \mathrm{x}_{\mathrm{i}+1}-\left(\Sigma_{1} \mathrm{x}_{\mathrm{i}}\right)\left(\Sigma_{2} \mathrm{x}_{\mathrm{i}}\right)\right] /\{[(\mathrm{n}-1) \\
\left.\left.\Sigma_{1} \mathrm{x}_{\mathrm{i}}^{2}-\left(\Sigma_{1} \mathrm{x}_{\mathrm{i}}\right)^{2}\right]^{1 / 2}\left[(\mathrm{n}-1) \Sigma_{2} \mathrm{x}_{\mathrm{i}}^{2}-\left(\Sigma_{2} \mathrm{x}_{\mathrm{i}}\right)^{2}\right]^{1 / 2}\right\},
\end{gathered}
$$

siendo $x_{i}$ cada elemento de la serie cronológica compuesta de $n$ términos. La sumatoria 1 abarca de $i=1$ a $i=n-1$ y la 2 , de $i=2$ a $i=n$.

Si la distribución de frecuencias de la serie se aproxima a la normal o gaussiana, el valor crítico de $r_{1}$ estará dado por

$$
\left(r_{1}\right)_{c}=\left[-1+Z(n-2)^{1 / 2}\right] /(n-1),
$$

donde, $Z$ es la desviación normal estándar de una cola al $95 \%$ de probabilidad, es decir, $Z=1.645$; si y solo si, $r_{1}$ es mayor que $\left(r_{l}\right)_{c}$, se concluye que la serie es no aleatoria, inconsistente o que sus términos no son independientes. Cuando llegan a ocurrir valores negativos de $r_{1}$, se debe a que la serie tiene una oscilación marcada; entonces es preferible usar una prueba de dos colas $(Z=1.960)$ y se debe sustituir el signo más de la ecuación 22 por uno negativo (WMO,1971).

Cuando se construye un correlograma, gráfica que relaciona los retrasos $(k)$ en el eje de las abscisas contra los correspondientes coeficientes de correlación serial de orden $k$, su significancia se prueba a través del test gráfico de Anderson (Linsley et al.,1977)

\section{Referencias}

Campos A. (1988). Función de distribución de probabilidades Gamma Mixta: Soluciones y aplicaciones, en: Memoria del $10^{\circ}$ Congreso nacional de hidráulica, Tomo II, pp. 318-322. Morelia, Michoacán.
Campos A. (1991). Propuesta de criterios para la elaboración de estudios hidrológicos (contexto hidrológico regional). Ingeniería hidráulica en México, Vol. VI, No. 3, 23-40.

Campos A. (1992a). Procesos del ciclo hidrológico. Universitaria Potosina, San Luis Potosí, S.L.P.

Campos A. (1992b). Estudios de homogeneidad en 34 estaciones pluviométricas del altiplano potosino. XII Congreso Nacional de Hidráulica (AMH). Tema 5: Investigación y Tecnología, Puerto Vallarta, Jal., octubre 1992.

CFE. Comisión Federal de Electricidad (1970). Manual de diseño de obras civiles. CFE, Ed, México.

CNA. Comisión Nacional del Agua (1997). Banco Nacional de datos de aguas superficiales (BANDAS). Subdirección General Técnica. México.

Fiering M.B. (1963). Use of Correlation to Improve Estimates of the Mean and Variance. Geological Survey Professional Paper 434-C. U.S. Department of the Interior, Washington, D.C.

Linsley R.K., M.A. Kohler y J.L.H. Paulus (1977). Hidrología para ingenieros. Editorial McGraw-Hill Latinoamericana, Bogotá, Colombia.

Maisel L. (1973). Probabilidad y estadística. Fondo Educativo Interamericano. Bogotá, Colombia.

Ruiz-Maya L. (1977). Métodos estadísticos de investigación. Instituto Nacional de Estadística. Madrid, España.

SHR. Secretaría de Recursos Hidráulicos (1970). Jefatura de Irrigación y Control de Ríos. Boletín hidrológico No. 36. Región hidrológica No. 10.

SRH. Secretaría de Recursos Hidráulicos (1975). Actualización al boletín hidrológico No. 36. Región hidrológica No. 10. Sinaloa, periodo 1970-73. Subsecretaría de Planeación. México.

WMO. World Meteorological Organization (1971). Annexe III: Standard Tests of Significance to be Recommended in Routine Analysis of Climatic Fluctuations, pp. 58-71, in Climatic Change. Technical Note No. 79, WMO-No. 195. T.P. 100. Geneva, Switzerland. de Estudios de Posgrado de la Facultad de Ingeniería, UNAM le otorgó los títulos de maestro (1980); y doctor (1987) en ingeniería hidráulica y aprovechamientos hidráulicos, respectivamente.En 1989, obtuvo la medalla "Gabino Barreda" de la UNAM. Ha publicado en revistas nacionales como: Ingeniería hidráulica en México y Agrociencia. Asimismo, ha participado en congresos nacionales e internacionales. Actualmente, es investigador nacional y profesor de la División de Educación Continua en la Facultad de Ingeniería, UNAM. 